Journal for ImmunoTherapy of Cancer

\title{
Real-world experience with elective discontinuation of PD-1 inhibitors at 1 year in patients with metastatic melanoma
}

\author{
Rebecca Pokorny, ${ }^{1}$ Jordan P McPherson, ${ }^{1}$ Benjamin Haaland, ${ }^{2}$ \\ Kenneth F Grossmann, ${ }^{3}$ Carolyn Luckett, ${ }^{3}$ Benjamin Newell Voorhies, ${ }^{4}$ \\ Daniel S Sageser, ${ }^{1}$ Jocelyn Wallentine, ${ }^{3}$ Zachary Tolman, ${ }^{1}$ Siwen Hu-Lieskovan, ${ }^{3}$ \\ Umang Swami ${ }^{3}$
}

To cite: Pokorny R,

McPherson JP, Haaland B, et al. Real-world experience with elective discontinuation of PD-1 inhibitors at 1 year in patients with metastatic melanoma. Journal for ImmunoTherapy of Cancer 2021;9:e001781. doi:10.1136/jitc-2020-001781

Preliminary results of this study have been presented as a poster at ASCO 2020 Virtual Annual Meeting on May 29, 2020.

Accepted 29 December 2020

Check for updates

(C) Author(s) (or their employer(s)) 2021. Re-use permitted under CC BY-NC. No commercial re-use. See rights and permissions. Published by BMJ.

1Department of Pharmacy, Huntsman Cancer Institute, University of Utah, Salt Lake City, Utah, USA

${ }^{2}$ Division of Oncology and Department of Population Health Sciences, Huntsman Cancer Institute, University of Utah, Salt Lake City, Utah, USA

${ }^{3}$ Department of Oncology, Huntsman Cancer Institute, University of Utah, Salt Lake City, Utah, USA

${ }^{4}$ Division of Oncology, Department of Medicine, Intermountain Healthcare, Salt Lake City, Utah, USA

Correspondence to Dr Umang Swami; umang.swami@hci.utah.edu

\section{ABSTRACT}

Background Randomized trials evaluating programmed cell death protein 1 (PD-1) inhibitors in metastatic melanoma either permitted treatment for 2 years (pembrolizumab) or more (nivolumab). The optimal duration of therapy is currently unknown due to limited data, and shorter therapies may be effective.

Methods Data of patients with metastatic cutaneous melanoma treated with single-agent PD-1 inhibitors at Huntsman Cancer Institute from January 1, 2015, to December 31,2018 , was reviewed to identify a continuous series of patients who made the joint decision with their provider to electively discontinue therapy at 1 year (>6 months and $<18$ months) in the setting of ongoing treatment response or disease stability. Patients were excluded if they received PD-1 inhibitors with other systemic therapy, had prior exposure to PD-1 therapy, or discontinued treatment due to disease progression or immune-related adverse event. Best objective response (BOR) per RECIST V.1.1 at treatment discontinuation, progression-free survival (PFS), and retreatment characteristics was analyzed.

Results 0 480 patients who received PD-1 inhibitors, 52 met the inclusion criteria. The median treatment duration from first to the last dose was 11.1 months $(95 \% \mathrm{Cl}$ 10.5 to 11.4). BOR was complete response in $13(25 \%)$, partial response in 28 (53.8\%), and stable disease in 11 (21.2\%) patients. After a median follow-up of 20.5 months (range 3-49.2) from treatment discontinuation, 39 (75\%) patients remained without disease progression, while 13 (25\%) had progression (median PFS 3.9 months; range 0.7-30.9). On multivariable analysis, younger age, history of brain metastasis, and higher lactate dehydrogenase at the time of anti-PD-1 discontinuation were associated with recurrence. Patients with recurrent melanoma were managed with localized treatment, anti-PD-1 therapies, and BRAF-MEK inhibitors. All patients except one were alive at data cutoff.

Conclusion In this large real-world, observational cohort study, the majority of patients with metastatic melanoma after 1 year of anti-PD-1 therapy remained without progression on long-term follow-up. The risk of disease progression even in patients with residual disease on imaging was low. After prospective validation, elective
PD-1 discontinuation at 1 year may reduce financial and immunotherapy-related toxicity without sacrificing outcomes.

\section{INTRODUCTION}

Since 2011, the US Food and Drug Administration has approved more than 10 treatment regimens for the treatment of advanced melanoma. ${ }^{1}$ These include antibodies targeting the programmed cell death protein-1 (PD-1) pathway, cytotoxic T-lymphocyte-associated protein 4 (CTLA-4), and inhibitors targeting BRAF mutations. ${ }^{2}$ The advent of these therapies has resulted in an improvement in overall survival (OS) as evidenced by a decrease in the overall mortality rate of patients with melanoma by $17.9 \%$ from 2013 to $2016 .{ }^{2}$ At present, two PD-1 inhibitors (pembrolizumab and nivolumab) are approved as single agents for the treatment of patients with metastatic melanoma. ${ }^{3-5}$ In randomized clinical trials, single-agent pembrolizumab and nivolumab have shown a median OS of 32.7-37.5 months. ${ }^{3-5}$

In the landmark clinical trials of PD-1 inhibitors in metastatic melanoma, the duration of pembrolizumab was limited to 2 years while nivolumab was allowed beyond 2 years. ${ }^{3-5}$ The optimal duration of anti-PD-1 therapies is currently unknown, and shorter courses may be as effective as prolonged courses for 2 or more years. ${ }^{67}$ In this study, we reviewed the clinical outcomes of patients who electively discontinued PD-1 inhibitors at 1 year at our institution.

\section{METHODS}

After Institutional Review Board approval, data of patients treated at the Huntsman 
Cancer Institute from January 1, 2015, to December 31, 2018, were retrieved from the electronic medical record. Inclusion criteria included $\geq 18$ years of age, a pathologically confirmed diagnosis of advanced cutaneous melanoma (unresectable stage III or stage IV), and treatment with single-agent anti-PD-1 therapy for 1 year ( $>6$ months and $<18$ months). The decision to discontinue anti-PD-1 therapy at 1 year was based on the joint decision with the treating oncologist and the patient in the setting of ongoing treatment response or disease stability. Exclusion criteria included a diagnosis of ocular or mucosal melanoma, receipt of anti-PD-1 therapy in the adjuvant or neoadjuvant setting, receipt of anti-PD-1 therapy in combination with other systemic or injectable therapies (eg, talimogene laherparepvec and ipilimumab), any prior treatment with PD-1 inhibitors, treatment on a clinical trial, or discontinuation of anti-PD-1 therapy due to treatment-related toxicity or progression.

Collected data included patient demographics, neutrophil-to-lymphocyte ratio (NLR), lactate dehydrogenase $(\mathrm{LDH})$, prior lines of treatment, duration of treatment, mutation status, and retreatment characteristics. We collected efficacy data consisting of best objective response (BOR) per RECIST V.1.1 at the time of anti-PD-1 discontinuation, progression-free survival (PFS), and OS.

Time to progression was computed from date of last PD-1 therapy to date of progression, subject to right censoring at last follow-up. Potential predictors of time to progression were screened via univariate Cox proportional hazards analyses. Potential predictors with $\mathrm{p}<0.05$ in univariate analyses were assessed in a multivariable model, along with a preliminary assessment of the multivariable model's ability to discriminate between earlier versus later or never progressing patients via concordance index. ${ }^{8}$ Evidence of interactions was assessed via Wald tests.

\section{RESULTS}

\section{Baseline characteristics}

Overall, 480 patients received single-agent anti-PD-1 therapy, of which 52 met the inclusion criteria. Figure 1 presents the flow diagram of patient selection and table 1 presents a comprehensive list of baseline characteristics of patients meeting the inclusion criteria. Of the 52 patients, $38(73.1 \%)$ were male and the median age at the start of treatment was 60 (range 19-83) years. Treatment consisted of pembrolizumab in $48(92.3 \%)$ and nivolumab in $4(7.7 \%)$ of the patients. Nineteen $(36.5 \%)$ of the patients received prior treatment for metastatic melanoma (table 1) and $17(32.7 \%)$ had a history of brain metastasis.

\section{Treatment and response}

Median treatment duration from first to the last dose was 11.1 (95\% CI 10.5 to 11.4) months. The BOR at treatment discontinuation was complete response (CR) in 13 (25\%), partial response (PR) in $28(53.8 \%)$, and stable

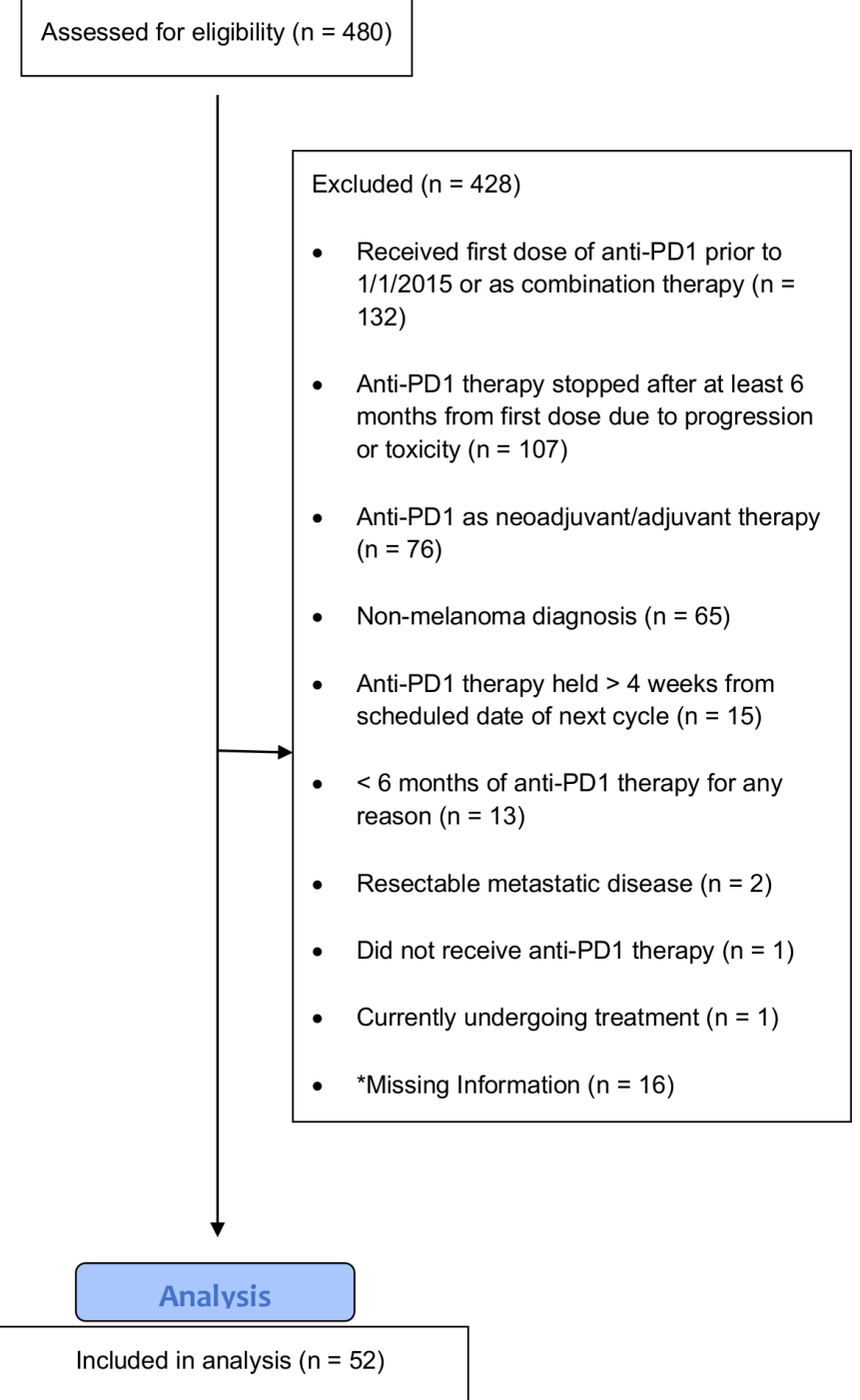

Figure 1 Flow diagram of patient selection. *Includes patients who received care at an outside facility or who were lost to follow-up. PD1, programmed cell death protein 1.

disease (SD) in $11(21.2 \%)$ patients. Figure 2 presents the Swimmer's plot summarizing treatment duration, the best response at the time of discontinuation of treatment, and time to progression. After a median follow-up of 20.5 (range 3-49.2) months after treatment discontinuation, $39(75 \%)$ patients remained without disease progression and the median PFS was not reached.

\section{Potential predictors of melanoma recurrence}

Univariate and multivariate analyses were conducted to evaluate potential predictors associated with melanoma recurrence. Analyzed variables included gender, age, history of brain metastasis, BOR, and NLR, and LDH prior to starting anti-PD-1 therapy and at the time of treatment discontinuation (last day of PD-1 therapy). Table 2 summarizes the univariate and multivariable results. Univariate analyses suggested that gender, age, history of brain metastasis at PD-1 initiation, and postPD-1 LDH may be associated with time to progression after 12-month PD-1 discontinuation. In multivariable 
Table 1 Baseline patient characteristics and comparison of relapsed and non-relapsed patients

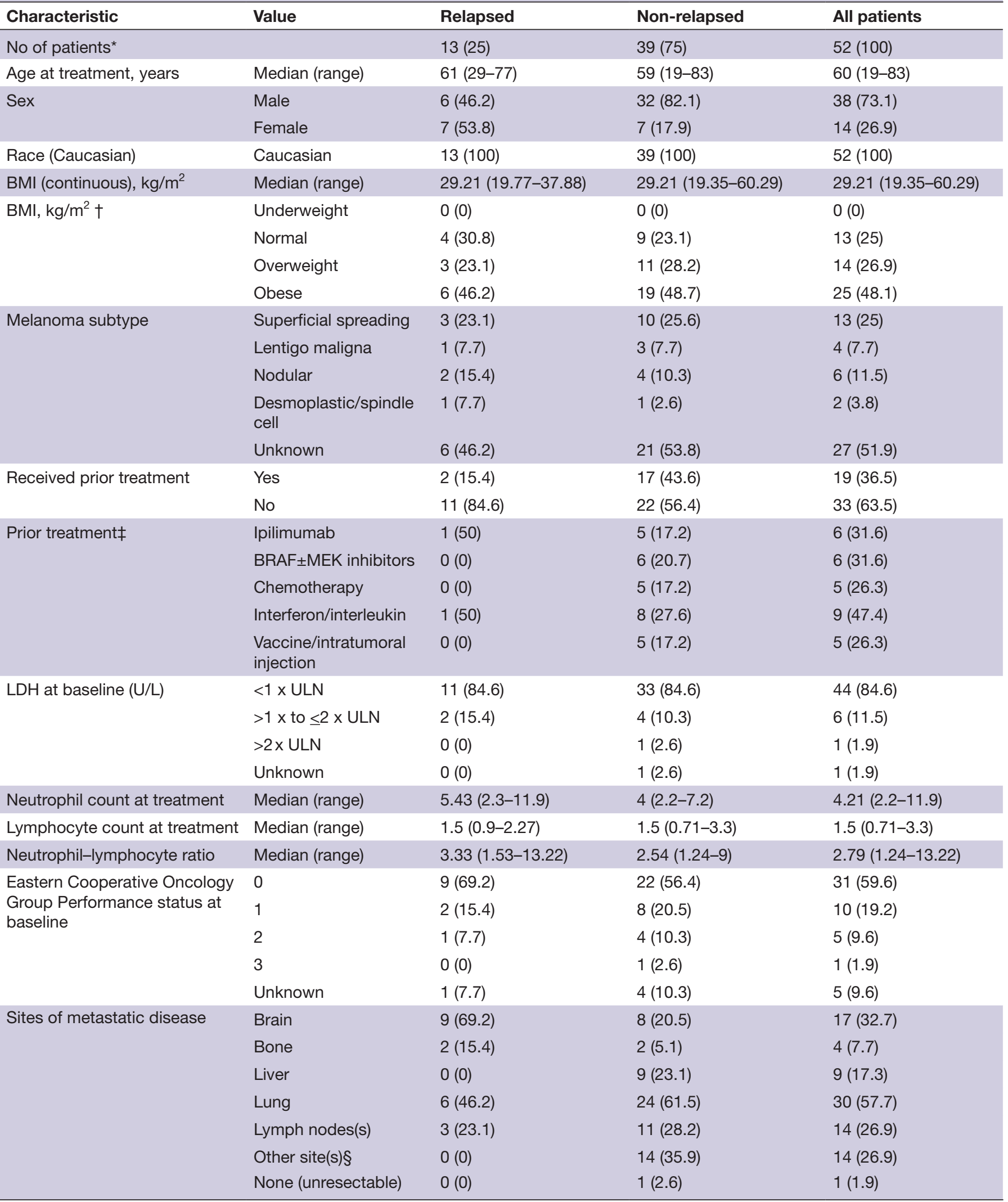

Continued 
Table 1 Continued

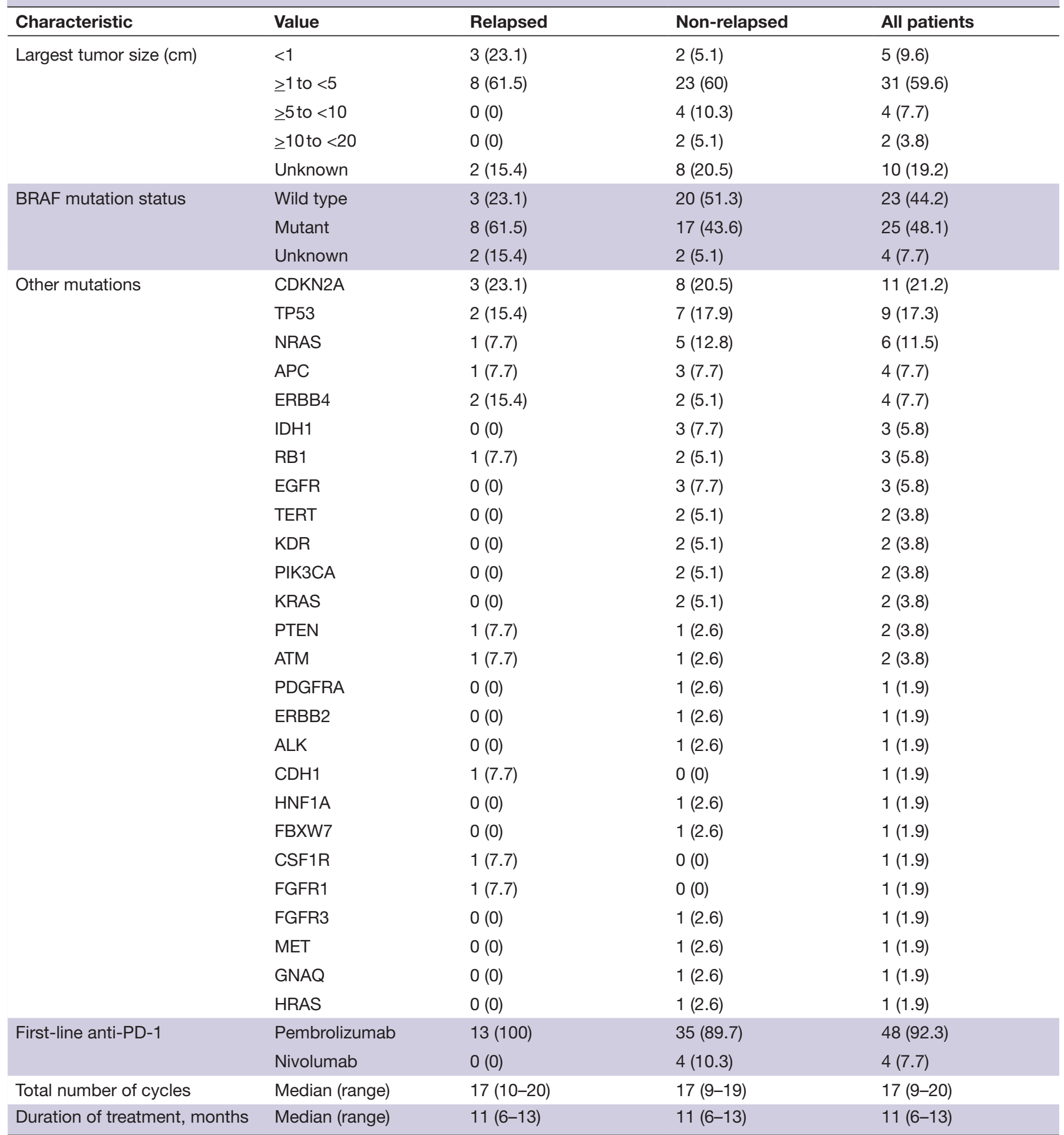

Data are no (\%) unless otherwise indicated.

*Per cent is row total, otherwise \% is column total.

†Underweight=BMI $<18.5 \mathrm{~kg} / \mathrm{m}^{2}$; normal weight=BMl $18.5-24.9 \mathrm{~kg} / \mathrm{m}^{2}$; overweight=BMI $25.0-29.9 \mathrm{~kg} / \mathrm{m}^{2} ;$ obese=BMI $\geq 30 \mathrm{~kg} / \mathrm{m}^{2}$.

†Per cent based on two patients (relapsed) and 17 (non-relapsed) who received prior treatment.

§Patients only counted once if they had one or multiple other sites of metastatic disease.

BMI, body mass index; LDH, lactate dehydrogenase; ULN, upper limit of normal.

analysis, these associations were slightly more attenuated and uncertain. There remained a strong evidence that younger age $(\mathrm{p}=0.037)$, history of brain metastasis $(\mathrm{p}=0.009)$, and greater post-PD-1 LDH $(\mathrm{p}=0.032)$ were associated with earlier time to progression. For fixed age, history of metastasis, and post-PD-1 LDH, there was no 


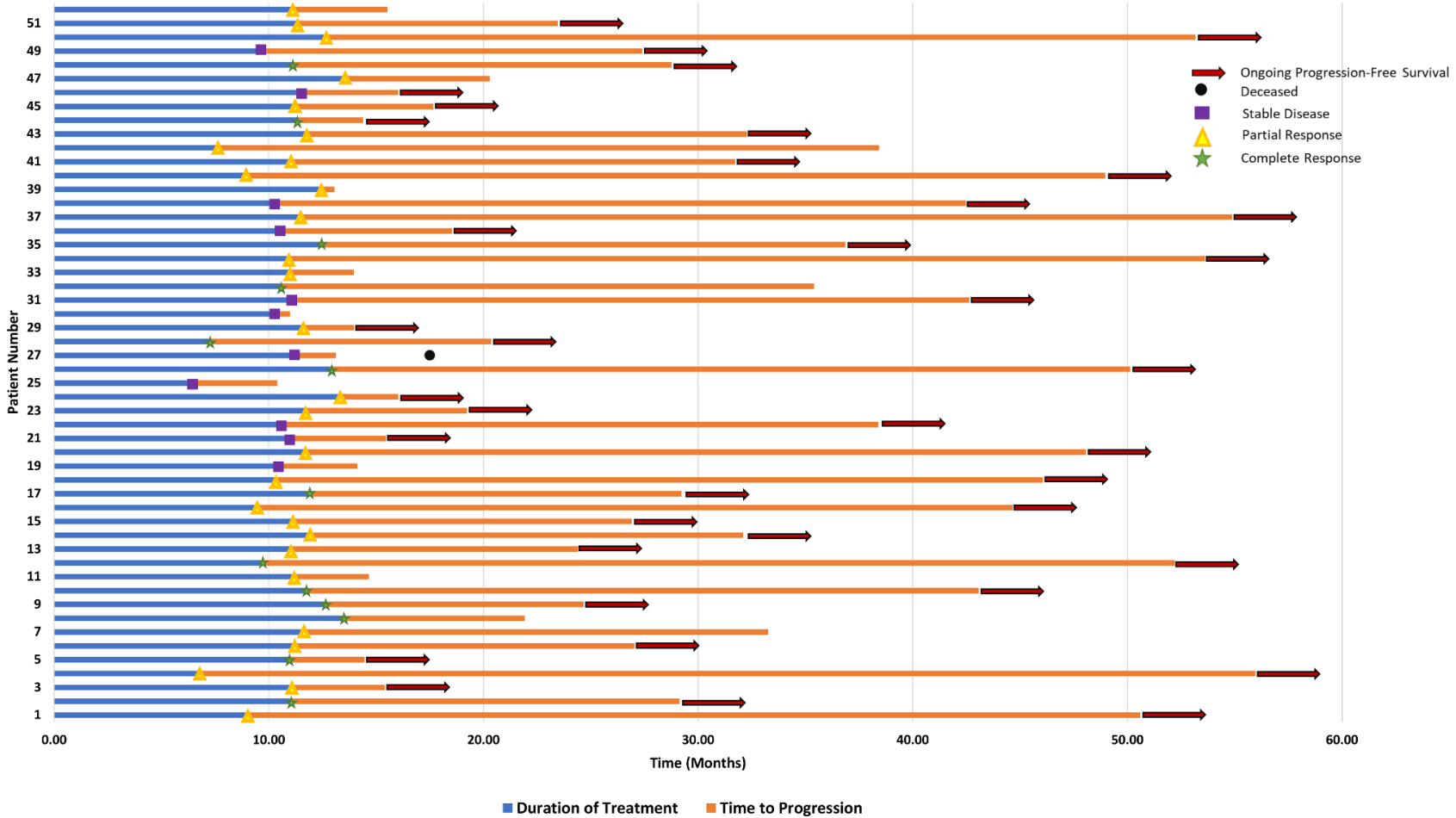

Figure 2 Swimmer's plot demonstrating duration of treatment, best response, and time to progression.

longer evidence that gender was associated with progression $(\mathrm{p}=0.228)$. For the multivariable model, the concordance index was 0.83 (95\% CI 0.70 to 0.95$)$, indicating the substantial ability of the multivariable model to discriminate between early versus later or never progressing patients. There were no statistically significant two-way interactions in the context of the multivariable model (all $\mathrm{p}>0.154)$.

\section{Relapse characteristics and retreatment patterns}

Thirteen $(25 \%)$ patients experienced disease progression. The median time to progression after treatment discontinuation was 3.9 (range 0.7-30.9) months. Of the relapsed patients two had CR, seven had PR and four had SD. Table 3 aggregates the relapse and treatment patterns and responses of these 13 patients.

Patients 1 and 2 had systemic multifocal failure but no metastasis to central nervous system (CNS). Both were retreated with anti-PD-1 therapy, which resulted in SD in patient 1 for more than 6 months and PR in patient 2. At data cutoff, both continued on anti-PD-1 therapy. Patient 3 had a solitary failure in the left adrenal and underwent adrenalectomy followed by observation only. Patient 4 had solitary progression in the right lung and underwent stereotactic body radiotherapy (SBRT). Seven months

Table 2 Univariate and multivariate analysis for evaluation of potential predictors associated with melanoma recurrence

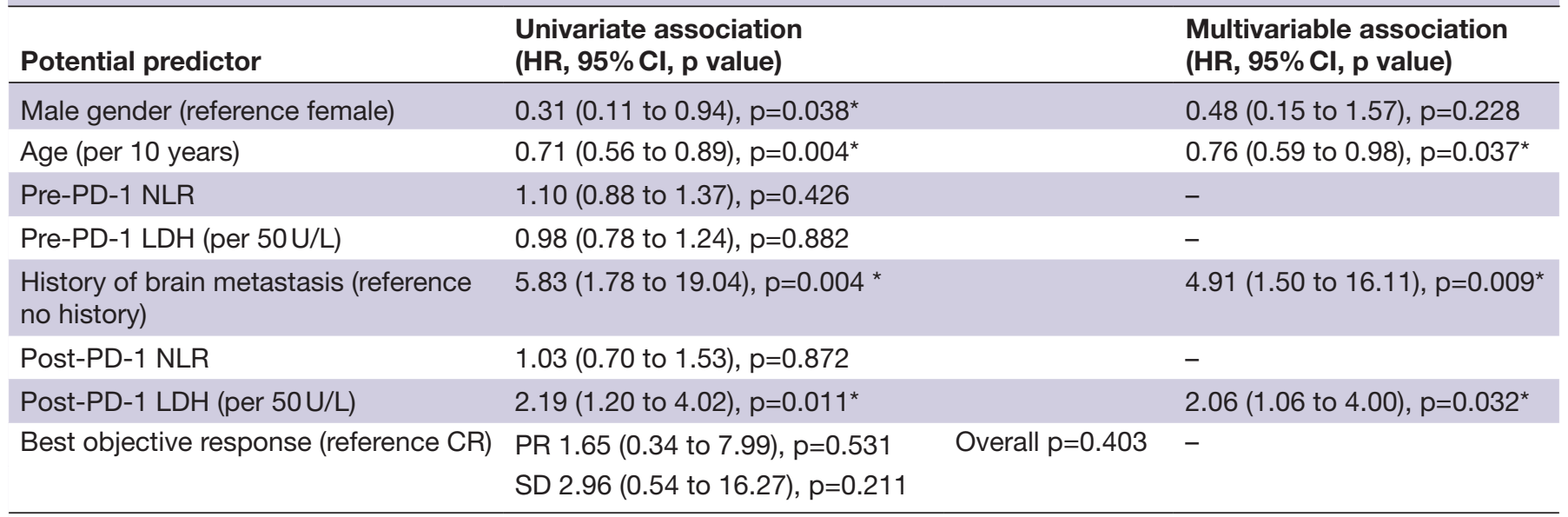

* $p$ values $<0.05$.

CR, complete response; LDH, lactate dehydrogenase; NLR, neutrophil-lymphocyte ratio; PD-1, programmed cell death protein 1; PR, partial response; SD, stable disease. 


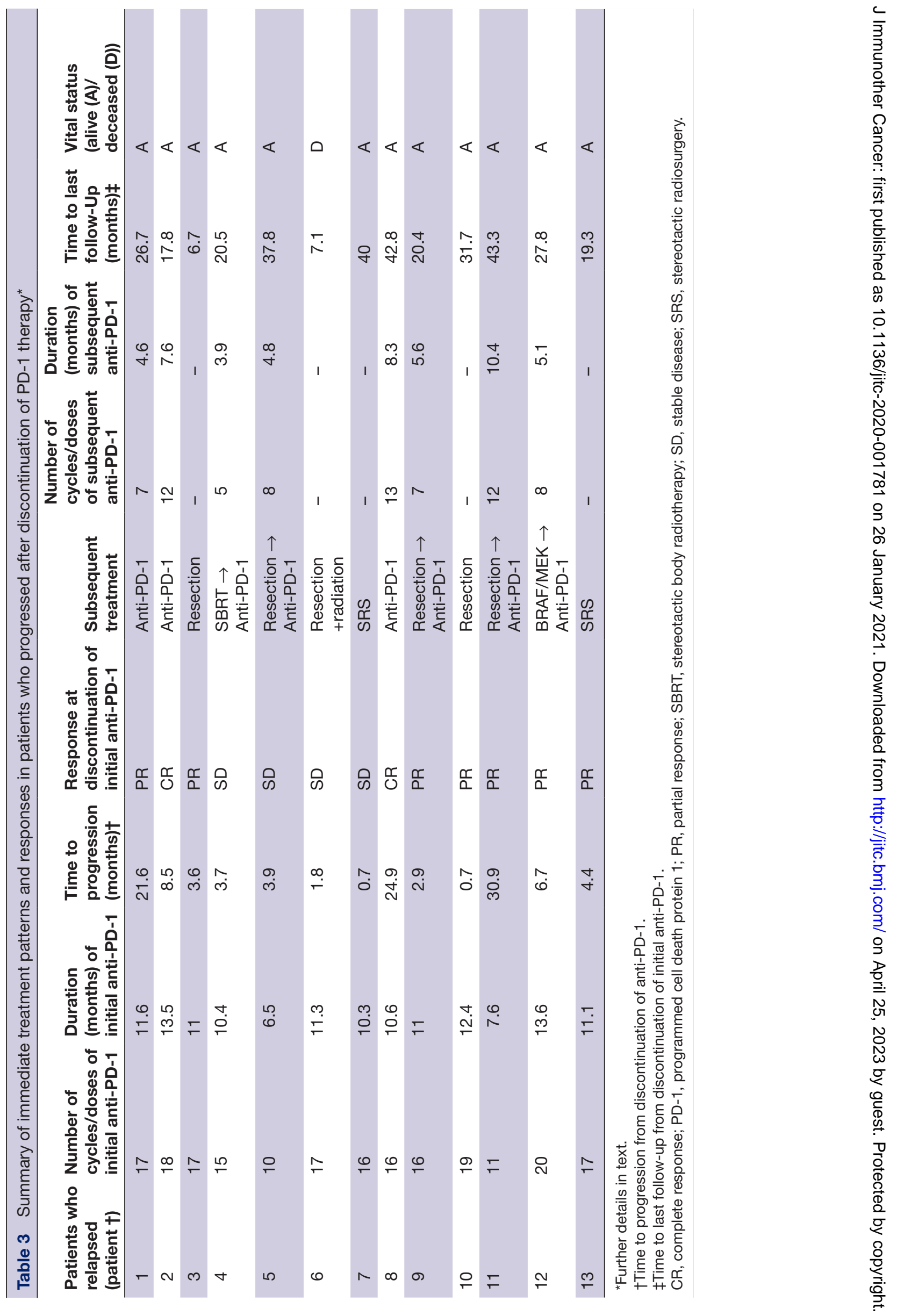


later, she had multifocal progression in the right lung and was started on anti-PD-1 therapy. After 3 months, she had progression in the brain, which was treated with stereotactic radiosurgery (SRS) and anti-PD-1 therapy was continued. Patient 5 had symptomatic progression in the brain and underwent resection of two brain lesions. Three months later patient was found to have systemic progression and was started on anti-PD-1 therapy, which led to SD for 6 months before progression needing splenectomy. Thereafter, the treatment was switched to nivolumab with ipilimumab. After four cycles patient had a mixed response and was switched to dabrafenib and trametinib, which caused PR. After 8 months, patient elected to switch back to anti-PD-1 therapy due to toxicities. Patient 6 was found to have progression in the brainstem. Since he did not have systemic progression outside CNS, he underwent resection and thereafter underwent postoperative radiation. Five months later, he had severe systemic progression and was transitioned to hospice. Patient 7 had CNS-only progression and underwent SRS and thereafter remained under observation. Patient 8 had multifocal progression in the left lung for which was started on antiPD-1 therapy. The patient had PR but had progression in brain lesions, which were treated with SRS and PD-1 therapy was continued. The patient subsequently discontinued therapy after 9 months, has CR, and is now under observation. Patient 9 had progression in the left adrenal, underwent adrenalectomy followed by adjuvant PD-1 therapy for 6 months. It was discontinued due to arthralgias and patient continues to be without any recurrence. Patient 10 had solitary progression in the lower lobe of the left lung for which he underwent left lower lobe resection followed by observation. Patient 11 had small bowel obstruction leading to resection followed by adjuvant PD-1, which continued till data cutoff without recurrence ( $>9$ months). Patient 12 had solitary progression in the right breast. The patient was started on encorafenib with binimetinib and after PR switched to anti-PD-1 therapy. Patient 13 had solitary progression in the brain, which was treated by SRS and since then is under observation.

In summary, relapse patterns include solitary non-CNS progression in six patients, CNS (brain) only progression in four patients, and multifocal non-CNS progression in three patients. Of these 13 patients, 9 immediately underwent successful localized treatment to the solitary site of progression, which included SRS or SBRT for 3 patients and surgical resection of the tumor for 6 patients. Of these nine patients, four were retreated with PD-1 inhibitors. Of the remaining four patients, three were retreated with PD-1 inhibitors and one received BRAF/MEK followed by PD-1 inhibitor. Retreatment with PD-1 inhibitor controlled the disease in all patients. All patients except one were alive at data cutoff.

\section{DISCUSSION}

Anti-PD-1 therapies are very effective for the treatment of metastatic melanoma. However, the optimal treatment duration is not well established. ${ }^{6}$ Continuing immunotherapies indefinitely predisposes patients to immune and financial toxicities and interferes with their quality of life. The adjusted total per patient per month cost for PD-1 inhibitors is approximately $\$ 13,059 .{ }^{9}$ Moreover, approximately $10 \%$ of patients who are treated with anti-PD-1 therapies develop grade 3 or higher treatment-related toxicities. ${ }^{10}$ These toxicities develop at different periods, with cutaneous toxicities such as rash, pruritus, and colitis developing earlier in the treatment course (within 8 weeks) and other toxicities such as acute interstitial nephritis manifesting much later (3-12 months). ${ }^{10} 11$ Delayed immune-related adverse events even after years of PD-1 therapies have been reported. ${ }^{12} \mathrm{~A}$ decrease in the duration of PD-1 therapies without impacting efficacy would therefore have a tremendous impact on the quality of life and financial health of these patients.

Our study presents one of the largest real-world, observational cohorts of patients who received antiPD-1 therapy outside of a clinical trial and made the informed decision to discontinue treatment at 1 year in the setting of disease stability and absence of immune toxicities. Prior evidence in this setting in melanoma is limited. In the multicohort phase 1 KEYNOTE001 trial, a protocol amendment allowed patients to discontinue pembrolizumab if they had a confirmed CR on two consecutive CT scans and at least 6 months of treatment, including at least two infusions after CR confirmation. ${ }^{13}$ In the 67 patients meeting the eligibility criteria and deciding to discontinue treatment, the 24 -month disease-free survival was $89.9 \% .^{13}$ In another real-world study, 185 patients with advanced melanoma discontinued treatment in the absence of treatment-limiting toxicities or progressive disease. ${ }^{14}$ The median duration of treatment was 12 months (range 0.7-43), and at the time of treatment discontinuation 117 (63\%) patients had CR, 44 (24\%) had PR, and $16(9 \%)$ patients had SD. At a median follow-up of 18 (range $0.7-48$ ) months after treatment discontinuation, $78 \%$ remained free of disease progression. The median time to progression was 12 (range 2-23) months, and progression was less frequent in patients who experienced CR (14\%) compared with patients with PR $(32 \%)$ and SD (50\%). ${ }^{14}$ Another study retrospectively evaluated 102 patients who achieved CR and discontinued treatment for any reason. ${ }^{15}$ The median duration of treatment was 9.4 (range, 1.6-36.1) months. The probability of treatment failure at 3 years was $27 \%$ and responses on retreatment were not common. Responses were seen only in 5 of 34 retreated patients with single-agent anti-PD-1 therapy and 11 of 44 patients with anti-PD-1 with anti-CTLA4-directed therapy. ${ }^{15}$ In a recent exploratory analysis of CheckMate 153, which evaluated continuous versus 1-year fixed-duration nivolumab in previously treated advanced non-small cell lung cancer patients, continuing nivolumab beyond 1 year appeared to be 
associated with improved outcomes. ${ }^{16}$ In the intent-totreat population, continuous nivolumab was associated with longer median OS compared with 1-year fixedduration treatment (not reached vs 28.8 months; HR, $0.62(95 \% \mathrm{CI}, 0.42$ to 0.92$)) .^{16}$

We conducted a multivariable analysis of baseline demographics and clinical variables to evaluate their association with progression. The results indicate that younger age, history of brain metastasis, and a higher post-PD-1 $\mathrm{LDH}$ were associated with earlier time to progression. These findings are not at all unexpected. In preclinical melanoma-bearing mice models, tumors in young mice were found to be more resistant to anti-PD-1 inhibition compared with older mice. This was attributed to a significantly higher population of regulatory $\mathrm{T}$ cells in young mice. ${ }^{17}$ In a multi-institutional cohort of 538 patients with metastatic melanoma, the odds of progressing on anti-PD-1 therapy decreased by $13 \%$ with each decade of patient age. ${ }^{17}$ In another study of 11,944 patients with metastatic melanoma, older ( $\geq 60$ years) compared with younger patients derived greater benefit with immunotherapies as evidenced by both multivariable Cox proportional hazards analysis and propensity score-weighted multivariable analysis. ${ }^{18}$ A similar difference in immunotherapy benefit based on age was not observed in other studies. ${ }^{19}{ }^{20}$ Elevated LDH is a surrogate marker of high tumor burden and an independent predictor of poor outcome. ${ }^{21} 22$ Similarly, brain metastasis is a known poor prognostic indicator with a historic median OS of 11.2 weeks. ${ }^{23}$ While intracranial response rates with combination therapies of ipilimumab and nivolumab and BRAF-MEK inhibitors range between $55 \%$ and $58 \%$ in patients with asymptomatic brain metastasis, responses are poor in patients with symptomatic brain lesions and with single-agent anti-PD-1 therapies. ${ }^{23}$

Our study has several important distinctions from previously reported studies. Our cohort not only included patients who had CR but also included patients with PR or SD. Treatment duration was restricted to 1 year based on our established institutional practice, in order to generate interpretable results for early treatment discontinuation. All our patients received treatment outside of clinical trial, making it a representative real-world cohort encountered in daily practice. No one discontinued treatment due to toxicity. Limitations include single institution, retrospective design, and lack of PD-L1 and tumor mutational burden analysis, which could have provided more insight with regard to reasons for non-durable responses. The multivariate model must be interpreted cautiously. In particular, the selective inference reported for the multivariate model after variable selection is expected to be slightly optimistic (ie, the actual type I error rates are expected to be slightly greater than the nominal $p$ values).

Our study presents preliminary evidence for the feasibility of early stopping of anti-PD-1 therapies at 1 year in patients with metastatic melanoma. Patients with younger age, brain metastasis, and greater post-PD-1 LDH may be at higher risk of relapse following early anti-PD-1 therapy discontinuation, and thus may not be ideal candidates for this practice. Randomized clinical trials such as STOP-GAP (NCT02821013) and DANTE (ISRCTN15837212) are evaluating optimal anti-PD-1 treatment duration and the role of rechallenge in metastatic melanoma. However, further studies are also needed with regard to the role of the tumor and immune biomarkers, circulating tumor DNA, and positron emission tomography-computed tomography imaging to help individualize the selection process for patients in whom a shorter treatment duration may be effective.

Acknowledgements The authors would like to acknowledge Ms Mingyuan Zhang and UHealth Data Science Services for their contribution to cohort identification.

Contributors Acquisition of data: RP, JPM, US. Analysis and interpretation of data: RP, JPM, BH, US. Review and revision of the manuscript: RP, JPM, BH, KFG, CL, BNV DSS, JW, ZT, SH-L, US. Writing, review, and/or revision of the manuscript: RP, JPM, BH, KFG, CL, BNV, DSS, JW, ZT, SH-L, US. Conception and design: JPM and US. Development of methodology: RP, JPM, BH and US.

Funding Research reported in this publication utilized the Cancer Biostatistics Shared Resource at Huntsman Cancer Institute at the University of Utah and was supported by the National Cancer Institute of the National Institutes of Health under Award Number P30CA042014.

Disclaimer The content is solely the responsibility of the authors and does not necessarily represent the official views of the $\mathrm{NIH}$.

Competing interests RP reports financial interest in Immunogen. BH reports consulting to AstraZeneca, Prometic Life Sciences, Value Analytics Labs, and the National Kidney Foundation. SH-L reports consulting to Amgen, Merck, Genmab, Xencor, BMS, research support from BMS, Merck, Vaccinex and contracted research from Neon Therapeutics, Astellas, F Star, Xencor, Merck, Vedanta and Boehringer Ingelheim. Other authors do not report any relevant conflicts of interest.

Patient consent for publication Not required.

Ethics approval The study was approved by Institutional Review Board at University of Utah.

Provenance and peer review Not commissioned; externally peer reviewed. Data availability statement Data are available upon reasonable request.

Open access This is an open access article distributed in accordance with the Creative Commons Attribution Non Commercial (CC BY-NC 4.0) license, which permits others to distribute, remix, adapt, build upon this work non-commercially, and license their derivative works on different terms, provided the original work is properly cited, appropriate credit is given, any changes made indicated, and the use is non-commercial. See http://creativecommons.org/licenses/by-nc/4.0/.

\section{ORCID iD}

Umang Swami http://orcid.org/0000-0003-3518-0411

\section{REFERENCES}

1 United States Food and Drug Administration. Hematology/ Oncology (Cancer) Approvals \& Safety Notifications. Available: https://www.fda.gov/drugs/resources-information-approveddrugs/hematologyoncology-cancer-approvals-safety-notifications [Accessed 23 Jun 2020].

2 Berk-Krauss J, Stein JA, Weber J, et al. New systematic therapies and trends in cutaneous melanoma deaths among US whites, 19862016. Am J Public Health 2020;110:731-3.

3 Larkin J, Chiarion-Sileni V, Gonzalez R, et al. Five-Year survival with combined nivolumab and ipilimumab in advanced melanoma. $N$ Engl J Med 2019;381:1535-46.

4 Ascierto PA, Long GV, Robert C, et al. Survival outcomes in patients with previously untreated BRAF wild-type advanced melanoma treated with nivolumab therapy: three-year follow-up of a randomized phase 3 trial. JAMA Oncol 2019;5:187-94.

5 Robert C, Ribas A, Schachter J, et al. Pembrolizumab versus ipilimumab in advanced melanoma (KEYNOTE-006): post-hoc 5-year 
results from an open-label, multicentre, randomised, controlled, phase 3 study. Lancet Oncol 2019;20:1239-51.

6 Seth R, Messersmith H, Kaur V, et al. Systemic therapy for melanoma: ASCO guideline. J Clin Oncol 2020;38:JCO2000198:3947-70.

7 National Comprehensive Cancer Network. Cutaneous melanoma (version 2.2020). Available: https://www.nccn.org/professionals/ physician_gls/pdf/cutaneous_melanoma.pdf [Accessed $23 \mathrm{Apr}$ 2020].

8 Harrell FE, Lee KL, Califf RM, et al. Regression modelling strategies for improved prognostic prediction. Stat Med 1984;3:143-52.

9 Klink AJ, Chmielowski B, Feinberg B, et al. Health care resource utilization and costs in first-line treatments for patients with metastatic melanoma in the United States. J Manag Care Spec Pharm 2019;25:869-77.

10 Martins F, Sofiya L, Sykiotis GP, et al. Adverse effects of immunecheckpoint inhibitors: epidemiology, management and surveillance. Nat Rev Clin Oncol 2019;16:563-80.

11 Wanchoo R, Karam S, Uppal NN, et al. Adverse renal effects of immune checkpoint inhibitors: a narrative review. Am J Nephrol 2017:45:160-9.

12 Couey MA, Bell RB, Patel AA, et al. Delayed immune-related events (DIRE) after discontinuation of immunotherapy: diagnostic hazard of autoimmunity at a distance. J Immunother Cancer 2019;7:165.

13 Robert C, Ribas A, Hamid O, et al. Durable complete response after discontinuation of pembrolizumab in patients with metastatic melanoma. J Clin Oncol 2018;36:1668-74.

14 Jansen YJL, Rozeman EA, Mason R, et al. Discontinuation of antiPD-1 antibody therapy in the absence of disease progression or treatment limiting toxicity: clinical outcomes in advanced melanoma. Ann Oncol 2019;30:1154-61.
15 Betof Warner A, Palmer JS, Shoushtari AN, et al. Long-term outcomes and responses to retreatment in patients with melanoma treated with PD-1 blockade. J Clin Oncol 2020;38:1655-63.

16 Waterhouse DM, Garon EB, Chandler J, et al. Continuous versus 1-year Fixed-Duration nivolumab in previously treated advanced non-small-cell lung cancer: CheckMate 153. J Clin Oncol 2020;38:JCO2000131:3863-73.

17 Kugel CH, Douglass SM, Webster MR, et al. Age correlates with response to anti-PD1, reflecting age-related differences in intratumoral effector and regulatory T-cell populations. Clin Cancer Res 2018;24:5347-56.

18 Jain V, Hwang W-T, Venigalla S, et al. Association of age with efficacy of immunotherapy in metastatic melanoma. Oncologist 2020;25:e381-5.

19 Elias R, Giobbie-Hurder A, McCleary NJ, et al. Efficacy of PD-1 \& PD-L1 inhibitors in older adults: a meta-analysis. J Immunother Cancer 2018;6:26.

20 Nishijima TF, Muss HB, Shachar SS, et al. Comparison of efficacy of immune checkpoint inhibitors (ICls) between younger and older patients: a systematic review and meta-analysis. Cancer Treat Rev 2016;45:30-7.

21 Balch CM, Soong SJ, Gershenwald JE, et al. Prognostic factors analysis of 17,600 melanoma patients: validation of the American joint Committee on cancer melanoma staging system. J Clin Oncol 2001;19:3622-34.

22 Weide B, Elsässer M, Büttner P, et al. Serum markers lactate dehydrogenase and $\mathrm{S} 100 \mathrm{~B}$ predict independently disease outcome in melanoma patients with distant metastasis. $\mathrm{Br} \mathrm{J}$ Cancer 2012;107:422-8.

23 Hong AM, Waldstein C, Shivalingam B, et al. Management of melanoma brain metastases: evidence-based clinical practice guidelines by cancer Council Australia. Eur J Cancer 2021;142:10-17. 